\title{
3D Visualization of Radar Backscattering Diagrams Based on OpenGL
}

\author{
Yulia V. Zhulina \\ Interstate Joint Stock Corporation “Vympel," P.O. Box 83, Moscow 1010001, Russia \\ Email:yulia_julina@mtu-net.ru \\ Received 20 May 2003; Revised 13 October 2003; Recommended for Publication by Xiang-Gen Xia
}

\begin{abstract}
A digital method of calculating the radar backscattering diagrams is presented. The method uses a digital model of an arbitrary scattering object in the 3D graphics package "OpenGL" and calculates the backscattered signal in the physical optics approximation. The backscattering diagram is constructed by means of rotating the object model around the radar-target line.
\end{abstract}

Keywords and phrases: radar scattering, backscattering diagrams, physical optics.

\section{INTRODUCTION}

The task, which represents a constant interest in radiolocation, is constructing a scattering diagram of an object illuminated by a radar. This task has been resolved more than once by the accurate mathematical methods of electrodynamics $[1,2,3]$, by experimental modeling of the radar systems $[4,5]$, by digital modeling of irradiated objects as combination of elements with known backscattering diagrams (such as pieces of the flat plates) $[6,7,8,9,10]$. The various methods for electromagnetic field modeling are comprehensively summarized in [11]. A review of generalized moment methods in the differential equations of electromagnetics is given in [12].

At present, the first method widely uses the numerical solution of the problem. Digital electromagnetics simulation allows accurate modeling of physical systems in combination with an accurate numerical solution of either differential or integral formulations of Maxwell's equations. This computational electromagnetics can be applied to many practical engineering problems, for example, antenna design, calculation, backscattering diagrams, targets recognition, and so forth. However, the accurate analysis and synthesis of complex electromagnetic systems have remained beyond the limits of computer capabilities up to now.

The most reliable approach has always been a method of natural modeling. It means that the model of object, constructed of real metallic or other materials, is irradiated by a real radar transmitter, and a real radar receiver gets the scattered signals. In this case, the very important thing is to keep the necessary proportions between the irradiated wavelength, the sizes of the object model, and the distance be- tween the radar and the object. These conditions are not always feasible. Besides, the natural and analog modeling are rather expensive and available for big organizations such as the White Sands Missile Range (WSMR), which possesses a highly specialized range instrumentation, technical laboratories, and facilities to support the continuing testing of NASA foreign and commercial systems. The modeling capabilities of the complex include tools for performing electromagnetic analysis, simulating electromagnetic wave propagation, and calculating antenna patterns. By the way, NASA publishes data of experimental measurements of radar cross sections for different shapes of radar targets [13].

Another and one of the most economical methods is modeling objects as the combination of elements such as pieces of the flat plates $[8,9,10,14]$. In [8], this modeling is used for simulating aircrafts and is performed in two steps. First, digital models are generated by the aircraft simulator tool. It provides information about the real shape and dimension of an aircraft. Aircraft surfaces are described by means of the following geometrical primitives: cylinder, frustum of cone, parallelepipeds, dihedral, and trihedral. Then this model (file description) is used to perform the second step of the model: a flat plates description of the aircraft. It uses three-dimensional (3D) representation and each small flat plate is characterized by its position and orientation with respect to the aircraft reference system. Each flat plate is an elementary scattering center that provides contribution to the signal echo received by radar. Physical optics theory of backscattering is used in order to simulate real signal. Although this approach can be realized by modern computers, it demands hard programmer efforts to construct every new object model. Recently, attempts have been made to apply 
the element approach to curved patches [15] although most of the works employ flat piecewise representation.

In this article, the method which represents the digital modeling of an object with the use of 3D graphics package "OpenGL" is proposed. This method simulates a scalar electromagnetic radiation of the object, but this task may be modeled also in the case of the polarized irradiation.

\section{THE FORMULA DESCRIPTION OF THE SCATTERED SIGNAL}

In the approximation of physical optics, the complex amplitude of a signal, reflected by a target and received by the radar position, disposed in the point $\vec{R}_{0}$, can be described by the Kirchhoff integral $[16,17,18]$. An approximate derivation of the Kirchhoff integral theorem is given in the appendix. If the locations of the transmitter and the receiver coincide $\left(\vec{R}_{0}=\vec{R}_{1}\right)$, the complex amplitude of the received signal can be described by the formula (up to a constant complex multiplier)

$$
\begin{aligned}
A\left(\vec{R}_{0}\right)=\int & \delta(F(\vec{r})) \frac{A_{0}}{\left|\vec{r}-\vec{R}_{0}\right|^{2}} \exp \left(-j \frac{4 \pi}{\lambda}\left|\vec{r}-\vec{R}_{0}\right|\right) \eta \\
& \times\left(-\vec{n}(\vec{r}) \cdot \frac{\left(\vec{r}-\vec{R}_{0}\right)}{\left|\vec{r}-\vec{R}_{0}\right|}\right) d^{3} \vec{r} .
\end{aligned}
$$

Here $\delta(x)$ is the delta function, $\vec{r}$ is the coordinate vector of the point of the object,

$$
F(\vec{r})=0
$$

is the equation of the surface of object,

$$
\eta(x)= \begin{cases}x, & \text { if } x \geqq 0, \\ 0, & \text { if } x<0,\end{cases}
$$

$A_{0}$ is the complex amplitude of the radar irradiation, $\left|\vec{r}-\vec{R}_{0}\right|$ is the distance from the point $\vec{r}$ to the point of the radar position $\vec{R}_{0}, \lambda$ is the length of the wave of the radar irradiation, and $\vec{n}(\vec{r})$ is the vector of the exterior normal to the surface of the object in the point $\vec{r}$. For the visible part of object $-\vec{n}(\vec{r}) \cdot\left(\left(\vec{r}-\vec{R}_{0}\right) /\left|\vec{r}-\vec{R}_{0}\right|\right)>0$ always.

If we could model the integral (1) in the various aspects of an object to the radar, we would get the scattering diagram of the object illuminated by the radar with wavelength $\lambda$.

\section{DESCRIPTION OF THE MODEL}

Practically, any 3D object can be modeled in the package OpenGL. This package allows to build spheres, cylinders, cones, prisms, polygons, and lines. Most complex objects can be constructed of all these elements. The every point of the surface of the constructed object has a $3 \mathrm{D}$ description in the

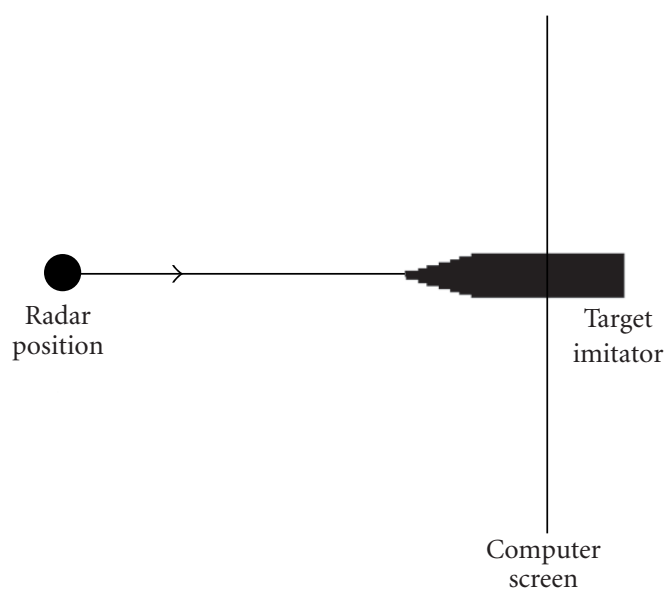

FIgUre 1: The whole scene of the model.

package: a two-dimensional (2D) position of the point on the computer screen $(X, Y)$ and the distance of the point to the screen $(Z$ coordinate). The object can be rotated relatively in any point in the space in an arbitrary way. It can be irradiated by the source of illumination disposed in an arbitrary space point. All these program properties permit to exactly simulate the radar system practically. And this package is suitable enough for calculating the backscattering diagram of the objects with various shapes. The calculation procedure is as follows.

(1) The object is placed on the screen plane and the radar irradiates the object in the direction perpendicular to the screen.

The irradiating scene and the model elements are shown in Figure 1. The position of the center mass of the object will be designed as $\vec{r}_{0}$, and this point $\vec{r}_{0}$ lies in the screen (in the center of the window which is to be processed). For each real point of the object $\vec{r}$, we will introduce the screen coordinates

$$
\vec{r}^{\prime}=\vec{r}-\vec{r}_{0} .
$$

(2) Every point of the object $\vec{r}^{\prime}$, visible in the screen, has coordinates $(X, Y)$ in the screen and coordinate $Z$ (its distance from the screen).

(3) Every point of the object $\vec{r}^{\prime}$, disposed on its surface and visible in the screen, gives a complex scattered amplitude $A\left(\vec{r}^{\prime}\right)$ in the point of radar $\vec{R}_{0}$ :

$$
\begin{aligned}
A\left(\vec{r}^{\prime}\right) \approx & \frac{A_{0}}{\left|\vec{r}_{0}-\vec{R}_{0}\right|^{2}} \exp \left(-j \frac{4 \pi}{\lambda}\left|\vec{r}^{\prime}+\vec{r}_{0}-\vec{R}_{0}\right|\right) \\
& \times \eta\left(-\vec{n}\left(\vec{r}^{\prime}\right) \cdot \frac{\left(\vec{r}_{0}-\vec{R}_{0}\right)}{\left|\vec{r}_{0}-\vec{R}_{0}\right|}\right),
\end{aligned}
$$

where all designations of the values are the same as in (1), (2b), and (2c). 
In the process of modeling, we will suppose the vector $\vec{r}-\vec{R}_{0}$ as follows:

$$
\vec{r}_{0}-\vec{R}_{0}=\left|\vec{r}_{0}-\vec{R}_{0}\right| \vec{e}
$$

where $\vec{e}=(0,0,1)$ is the normalized vector of the direction of the radar irradiation, directed perpendicularly to the screen.

The multiplier $-\vec{n}\left(\vec{r}^{\prime}\right) \cdot\left(\left(\vec{r}_{0}-\vec{R}_{0}\right) /\left|\vec{r}_{0}-\vec{R}_{0}\right|\right)$ is created automatically in the process of the $3 \mathrm{D}$ object modeling, as the package OpenGL simulates 3D objects and takes into account the direction of light source irradiation. So, we can get the value of intensity $E(X, Y)$ of the screen pixel with the screen coordinates $(X, Y)$ :

$$
E(X, Y)=-\vec{n}\left(\vec{r}^{\prime}\right) \cdot \frac{\left(\vec{r}_{0}-\vec{R}_{0}\right)}{\left|\vec{r}_{0}-\vec{R}_{0}\right|} .
$$

For getting the visible part of the surface, the package calculates only values $E(X, Y)>0$. If the object is disposed in a far zone, the condition is true:

$$
\left|\vec{r}-\vec{r}_{0}\right|=\left|\vec{r}^{\prime}\right| \ll\left|\vec{r}_{0}-\vec{R}_{0}\right|,
$$

and we can write the distance of each point to the radar as follows:

$$
\left|\vec{r}-\vec{R}_{0}\right|=\left|\vec{r}^{\prime}+\vec{r}_{0}-\vec{R}_{0}\right| \approx Z_{0}+Z
$$

Here $Z_{0}=\left|\vec{r}_{0}-\vec{R}_{0}\right|$ is the distance from the screen to the radar and $Z$ is the distance of the point to the screen. So (3) can be rewritten as follows:

$$
A(X, Y, Z)=\frac{A_{0}}{Z_{0}^{2}} \exp \left(-j \frac{4 \pi}{\lambda}\left(Z+Z_{0}\right)\right) E(X, Y) .
$$

(4) We must summarize all the amplitudes (8) over the whole visible and illuminated part of the object surface to get the whole signal received by the radar from the range $Z$ :

$$
S(Z)=\frac{A_{0}}{Z_{0}^{2}} \exp \left(-j \frac{4 \pi}{\lambda}\left(Z+Z_{0}\right)\right) \sum_{i=1}^{I} \delta\left(Z_{i}, Z\right) E\left(X_{i}, Y_{i}\right) .
$$

In (9), the function $\delta\left(Z_{i}, Z\right)$ is as follows:

$$
\delta\left(Z_{i}, Z\right)= \begin{cases}1, & \text { if } Z_{i}=Z, \\ 0, & \text { if } Z_{i} \neq Z,\end{cases}
$$

$Z_{i}$ is the distance from the screen of the pixel with the screen coordinates $X_{i}, Y_{i}$ and $I$ is the whole summarized number of pixels in the visible part of the object surface.

(5) To get the signal $S_{0}$ received by the radar, we have to summarize the signals (9) over all the band of the range values and calculate the total amplitude:

$$
S_{0}=\sqrt{\left|\sum_{m=1}^{M} S\left(Z_{m}\right)\right|^{2}},
$$

where $M$ is the whole number of the discrete ranges in the band of ranges.

(6) Notice that the signal $S_{0}$ is the function of the angles of the object rotation relative to the radar, that is,

$$
S_{0}=\tilde{S}_{0}(\alpha, \beta, \gamma),
$$

where $\alpha, \beta$, and $\gamma$ are the angles of rotation around axes $Y, X$, and $Z$, correspondingly. If the object is an axissymmetrical body, then expression (12) is the function of the single angle $\alpha$ between the axis of symmetry and direction $\vec{e}$ (formula (4)) to the radar:

$$
S_{0}=\tilde{S}_{0}(\alpha) .
$$

In this case, the construction of the scattering diagram leads to the calculation of all the meanings (13) over the whole range of values $\alpha$. And this sequence is the scattering diagram.

\section{THE RESULTS OF THE DIGITAL MODELING}

The process of calculations consists of the following operations:

(1) rotating the object around the vertical axis $Y$ at the angle $\alpha$. In all the figures, the axis $Y$ is directed from the bottom to top;

(2) calculating the complex signal (8) from each pixel of the screen belonging to the visible part of the object;

(3) summarizing these signals from the pixels lying at the same distance $Z$ from the screen by formula (9). As a result, we get a coherent complex wideband signal from the object;

(4) accumulating signals of all ranges and calculating the amplitude of the signal by formula (11);

(5) recording the diagram for value $\alpha$;

(6) calculating the new angle $\alpha$ for the new object aspect;

(7) transfering to the point of calculations (1).

The results of modeling are shown in Figures 2, 3, 4, 5, 6, and 7 for the sphere, cylinder, cone, and object representing the combination of the cone and cylinder.

In all the figures, the relative position of the object to the screen (just the same as to the radar) is shown at the moment of getting the current point of the diagram. The graphic of the diagram is being drawn moving with a discrete of angle $\alpha$ equal to del $\alpha=0.5^{\circ}$ (Figures 2, 3, 4, 5, 6, and 7).

The initial angle $\alpha=45^{\circ}$ in all the figures and the meanings of $\alpha$ are decreasing (rotating over $y$-axis in the clockwise direction).

It is necessary to notice that all the dimensions of objects, pointed below, were used in the process of calculations, but these dimensions are not exactly supported in Figures 2, 3, 4, 


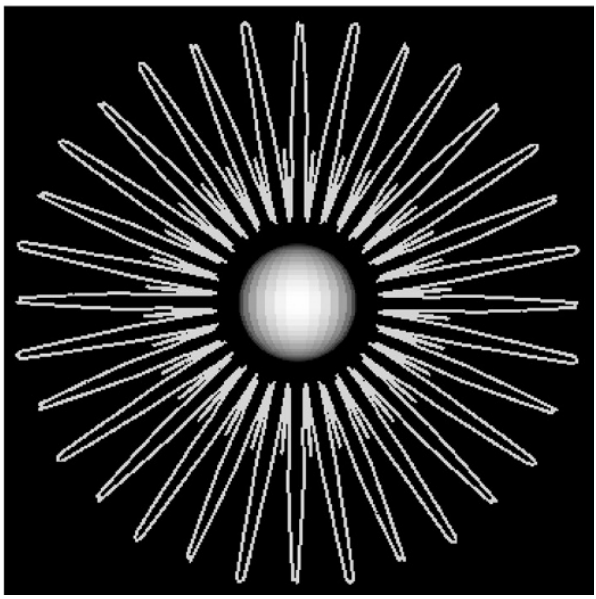

FIGURE 2: The whole scattering diagram of the sphere $\left(45^{\circ} \div 45^{\circ}-\right.$ $\left.360^{\circ}\right)$.

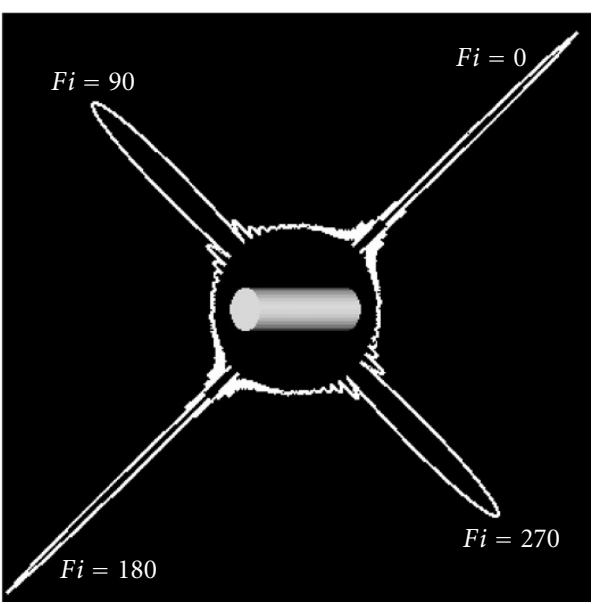

FIgURE 3: The whole scattering diagram of the cylinder $\left(45^{\circ} \div 45^{\circ}-\right.$ $360^{\circ}$.

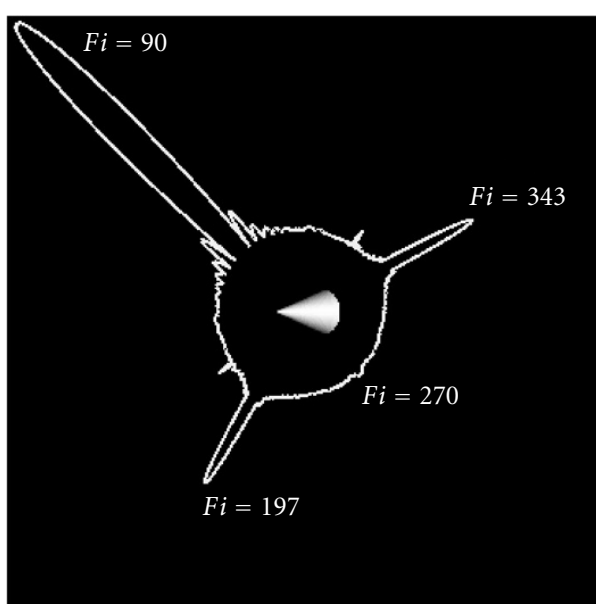

FIGURE 4: The whole scattering diagram of the cone $\left(45^{\circ} \div 45^{\circ}-\right.$ $\left.360^{\circ}\right)$.

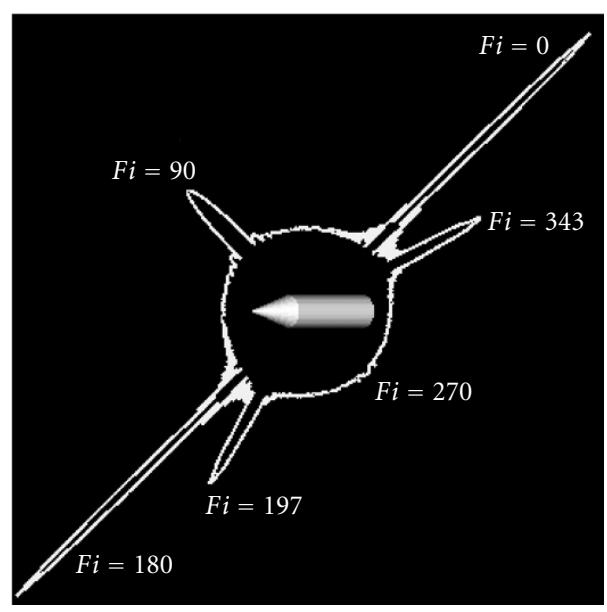

FIgure 5: The whole scattering diagram of the cone-cylinder composition $\left(45^{\circ} \div 45^{\circ}-360^{\circ}\right)$.

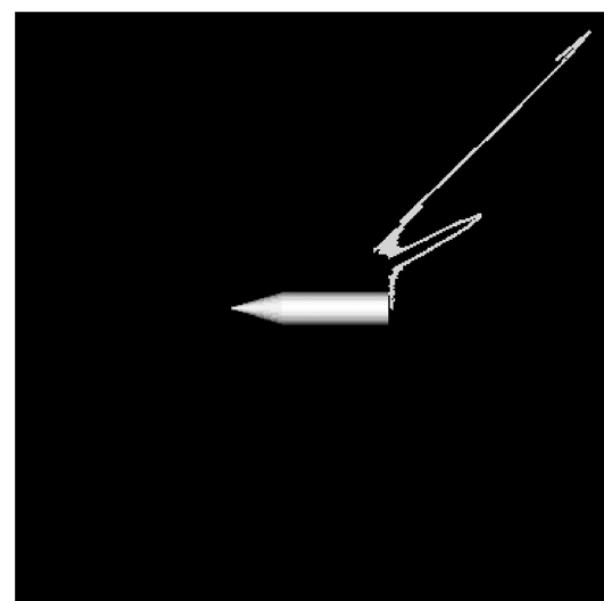

Figure 6: Signal in the direction of the normal to the surface of cylinder in the cone-cylinder composition.

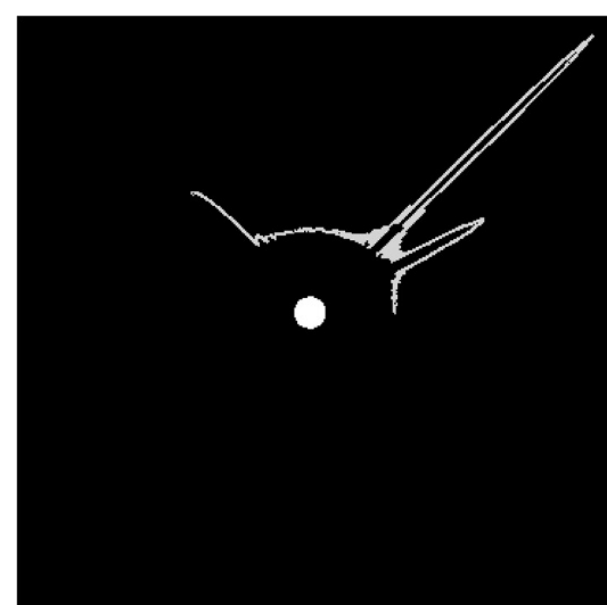

Figure 7: Signal from the bottom of the cylinder in the conecylinder composition. 
5,6 , and 7. Some of the objects are increased in sizes for the better visualization of the figures.

In Figures 3, 4, and 5, the angle values $F i$ are shown, which are equal to the corresponding value of angle $\alpha$ at the moment.

All the calculations have been performed in the windows with the dimension of $360 \times 360$ pixels in Figures 2, 3, and 4 , and in Figures 5, 6, and 7 in the window with the size of $400 \times 400$ pixels. The width of the single pixel was taken equal to $\lambda / 15.0$ in all the calculations.

Figure 2 shows the diagram from the sphere, which is constructed by 32 vertical slices. The radius of the sphere is equal to 50 pixels. Figure 2 shows the whole diagram when angle $\alpha$ becomes equal to $\alpha=45^{\circ}-360^{\circ}$ and the sphere is turned at the angle of $-360^{\circ}$.

From Figure 2, it is seen that the signal repeats itself 32 times round the $y$-axis. It matches the reflection from the 32 identical slices. If the number of slices streams to infinity, the sphere will be entirely smooth and its signal will be the same in all the directions.

Figure 3 shows the diagram of a cylinder. The cylinder is 200 pixels long and it has radius of 30 pixels. As the cylinder is an axis-symmetrical body, all the diagram is symmetrical relative to the main maximum. This maximum is received from the lateral surface of the cylinder as the lateral surface has the maximal radar cross section (in this example). This maximum is the narrowest among the others because the height of the cylinder is approximately 3 times more than the diameter of the bottom.

Two maximums of the second magnitude are produced by the bottom of the cylinder.

In Figure 3, the cylinder is turned to the angle of $-360^{\circ}$ from its start angle position $\alpha=45^{\circ}$.

Figure 4 shows the diagram of a cone. The cone is 100 pixels long and has a bottom radius of 30 pixels. As the cone is an axis-symmetrical body, all the diagram is symmetrical relative to the main maximum. This maximum is received from the bottom of the cone, as the bottom has the maximal radar cross section (in this example). This maximum is the widest among the others because the diameter of the bottom is $\sim 1.7$ times less than the height of the cone.

Two maximums of the second magnitude are produced by the flank of the cone when the direction to the radar receiver has the perpendicular angle to the flank surface of the cone. At this moment, $\alpha$ becomes equal to $\alpha=\beta$, where $2 \beta$ is an angle at the top of the cone and $\beta=\operatorname{arctg}(0.3) \approx 17^{\circ}$, and the cone is turned at the angle of $\beta-45^{\circ}=-28^{\circ}$.

The two more small maximums of the diagram are obtained when the visible part of the cone has the largest surface, but the normal is not directed to the radar receiver.

At last, the smallest maximum is provided by the nose of the cone from the direction opposite to the direction of the main maximum.

In Figure 4 , the cone is turned to the angle of $-360^{\circ}$ from its start angle position $\alpha=45^{\circ}$. Figures 5,6 , and 7 show the diagram from a cone plus a cylinder composition. The cylinder is 200 pixels long and has a radius of 30 pixels. The cone is 100 pixels long and has a bottom radius of 30 pixels. The

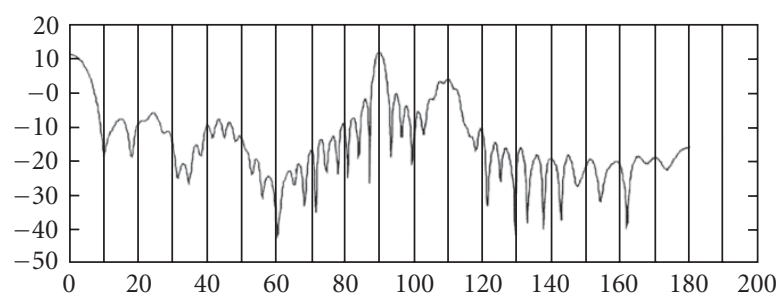

FIGURE 8: The scattering diagram of the cone-cylinder composition obtained by the method of flat plates.

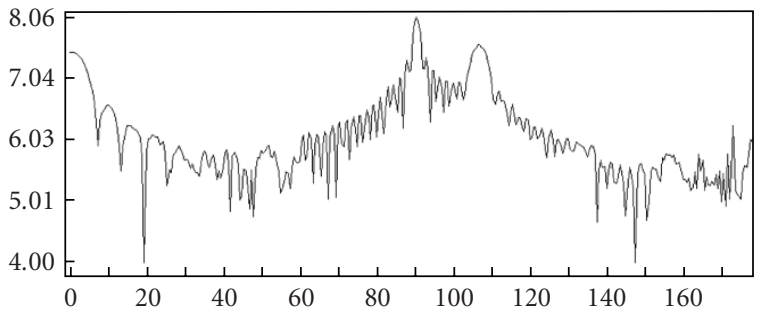

Figure 9: The scattering diagram of the cone-cylinder composition obtained by using OpenGL package.

composition is also the axis-symmetrical body and all the diagram (Figure 4) is symmetrical relative to the maximum received from the bottom of the cylinder.

The main maximum is given by the lateral surface of the composition at the moment when the direction to the radar receiver has the perpendicular angle to the lateral surface of the cylinder. All the construction has the maximal radar cross section in this aspect. This maximum is the narrowest among the others as the length of the construction is more than the diameter of the cylinder bottom.

Two maximums of the second magnitude are produced by the flank of the cone when the direction to the radar receiver has the perpendicular angle to the flank surface of the cone. The central maximum is provided by the bottom of the cylinder.

Figure 5 shows the whole diagram when angle $\alpha$ becomes equal to $45^{\circ}-360^{\circ}$ and the construction is turned at the angle of $-360^{\circ}$.

Figures 6 and 7 are given as an illustration of processing the diagrams. The drawings of the diagrams built until the moment and the aspect of the construction at the moment are shown. Figure 6 shows the maximum of the diagram from the lateral side of the cylinder when the direction to the radar receiver has the perpendicular angle to the lateral surface of the cylinder. At this moment, $\alpha$ becomes equal to $0^{\circ}$ and the whole construction is turned at the angle of $-45^{\circ}$.

Figure 7 shows the maximum of the diagram from the bottom of the cylinder.

Analysis of Figure 5 shows a good coincidence with analogue results obtained in [19] for analogue composition. In Figure 8, a diagram is shown, which was obtained by the method of flat plates for the cone-cylinder combination in 
[19]. Correspondingly, in Figure 9, the diagram of conecylinder (Figure 5) is unrolled in the angle space. The positions of maximums from the flank surface of the cone are different because the cone in Figure 8 has the angle at its top equal to $40^{\circ}$, and in Figure 9, it is equal to $34^{\circ}$. A ratio $\lambda /$ Dim, where Dim is a size of the whole object, is higher in Figure 8. That is why all the lobes in Figure 8 are wider than in Figure 9. The diagram in Figure 8 is given in $\mathrm{dB} / \mathrm{m}^{2}$; in other words, it is normalized to the cross section, and in Figure 9, it is given simply in logarithmic scale. But the whole pattern of the diagrams is the same.

There is a need to say that accurate building of diagrams with thorough drawing all the side lobes is achievable only when the ratio of each pixel width to the wave lambda $\lambda$ is small enough. In the calculations of the paper, this ratio was taken equal to $1 / 15$. So the accurate calculation of the large objects diagrams will demand the displays of large sizes.

\section{DISCUSSION}

The digital modeling of the physical optics integral (1) by the 3D graphics methods gives results (Figures 2, 3, 4, 5, 6, and 7) rather close to those obtained by the methods of flat places [19]. The described approach seems to be much less labor-intensive because it uses the ready-made 3D model of the object, and the algorithm only substitutes the coordinates and intensity of the screen pixels into the sum (9).

Using the flat places, approximation includes both the construction of an object and the calculation of (1). The whole volume of the works using this method can be estimated from $[8,9]$. Some steps have to be made: the first step is the definition of the whole set of the object flat plates; the next one is the analysis of elementary flat plates visible from the radar, and the calculation of the radar cross section of each scattering center of the object as function of flat plate orientation and position. After this, the complex echo signal received from each center and the total power of the echo signal for each radar resolution cell are calculated.

As it has been previously mentioned, the nature modeling may appear to be expensive or not available in the conditions of the theoretical laboratories.

As for the method of building backscattering diagrams by means of numerical solutions of Maxwell's equations, it should be mentioned that the equations are usually digitized with the help of a set of known expansion functions. This leads to a linear system of equations with $N$ unknowns, where $N$ is the number of expansion functions. In this case, the computer requirements are at least proportional to $\mathrm{N}^{2}$ in terms of both computer time and memory. Since $N$ may be very large, these requirements are beyond the capabilities of today's computers (e.g., 76 GB RAM if $N=100.000$ ) $[20,21]$.

To resolve the problem, researchers have developed the fast multipole method (FMM) and the multilevel fast multipole algorithm (MLFMA) [22, 23, 24]. It has been demonstrated that, using this MLFMA procedure, the calculations can be performed with order $N \log N$ complexity. Additionally, improved MLFMA approach is developed in [25]. This approach is more efficient computationally, especially as the number of $N$ increases.

In the presented method of modeling, the main computational requirements are imposed on the speed of reading pixels from the screen, in other words, on the speed of performing the OpenGL functions. The whole diagram of 720 points with reading all the pixels in the window of $400 \times 400$ pixels was calculated in 4.5 minutes at the computer with $256 \mathrm{MB}$ RAM and $1300 \mathrm{MHz}$ clock rate.

By the way, the method calculates the wideband signal in the process of building diagram (formula (9)), which can be used in a lot of radar simulation software.

So the presented results allow to draw a conclusion that the digital modeling of object with the use of $3 \mathrm{D}$ graphics package OpenGL gives opportunity of simulating electromagnetic radiation of the object. This method can reduce the workload of radar data simulation by using 3D graphics package constructed by many compilers. This method can be used by researchers who have no radar data simulation software.

The algorithm, proposed in this article, requires the presentation of the object with the dimensions of pixels much less than the length of wave $\lambda$ for the proper working. It is only the serious restriction on the method because a large object consists of large number of pixels and it will enlarge the time of calculations and will require a big display.

\section{APPENDIX}

The Fresnel-Kirchhoff approximation to diffraction is well known $[16,17,18]$. It is Fresnel diffraction principle that states that every unobstructed point of a wavefront, at a given instant time, serves as a source of spherical secondary waves (with the same frequency as that of the primary wave). The amplitude of the field at any point beyond is the superposition of all these waves (considering their amplitudes and phases). This rather hypothetical principle has been later on developed in a more rigorous way by Kirchhoff, who proved that it can be derived from the scalar diffraction theory.

Consider a radar transmitter and a receiving position disposed in the points $\vec{R}_{0}$ and $\vec{R}_{1}$, respectively.

The electromagnetic field at the receiver is a solution of the wave equation

$$
\nabla^{2} P=\frac{1}{c^{2}} \frac{\partial^{2} P}{\partial t^{2}}
$$

We can express the solution in the form $P(t, \vec{R})=\tilde{P}(\vec{R}) e^{j k c t}$ (where $c$ is the light speed, $k=2 \pi / \lambda$ is the wave number, $\lambda$ is the wavelength, $\omega=k c$ ). Substituting this in the wave equation, we obtain the Helmoltz equation:

$$
\nabla^{2} \tilde{P}+k^{2} \tilde{P}=0 .
$$

Solving this equation with the help of Green's theorem leads to an expression of the field at point $\vec{R}_{1}$ of the receiver in 
terms of the field and its gradient assigned on an arbitrary scattering surface $S$ enclosing $\vec{R}_{1}$ :

$$
\begin{aligned}
& \tilde{P}\left(\vec{R}_{1}\right)=\frac{1}{4 \pi}\left[\int_{S} \frac{e^{-j k\left|\vec{R}_{1}-\vec{r}\right|}}{\left|\vec{R}_{1}-\vec{r}\right|} \nabla_{\vec{n}(\vec{r})} \tilde{P}(\vec{r}) d S\right. \\
&\left.-\int_{S} \tilde{P}(\vec{r}) \nabla_{\vec{n}(\vec{r})}\left(\frac{e^{-j k\left|\vec{R}_{1}-\vec{r}\right|}}{\left|\vec{R}_{1}-\vec{r}\right|}\right) d S\right]
\end{aligned}
$$

which is known as the Kirchhoff integral theorem.

In (A.3), $\vec{r}$ designates the coordinates of the surface $S$ point, $d S$ is the differential of the surface, and $\nabla_{\vec{n}(\vec{r})} f(\vec{r})$ designates the gradient of $f(\vec{r})$ along the normal $\vec{n}(\vec{r})$ to the surface $S$ in the point $\vec{r}$. The boundary conditions $\left.\tilde{P}(\vec{r})\right|_{S}$ and $\left.\nabla_{\vec{n}(\vec{r})} \tilde{P}(\vec{r})\right|_{S}$ can be chosen as the values of the primary spherical wave irradiated by the transmitter and its gradient projections to the vector $\vec{n}(\vec{r})$, respectively.

The primary radiated spherical wave in the surface point $\vec{r}$ has the form

$$
P(t, \vec{r})=\frac{A}{\left|\vec{r}-\vec{R}_{0}\right|} e^{j\left(\omega t-k\left|\vec{r}-\vec{R}_{0}\right|\right)}=\tilde{P}(\vec{r}) e^{j \omega t},
$$

where

$$
\tilde{P}(\vec{r})=\frac{A}{\left|\vec{r}-\vec{R}_{0}\right|} e^{-j k\left|\vec{r}-\vec{R}_{0}\right|},
$$

$\left|\vec{r}-\vec{R}_{0}\right|$ is the distance from the transmitter in the point $\vec{R}_{0}$ to the point of the surface $\vec{r}$, and $A$ is the wave amplitude at the unit distance from the transmitter.

By direct calculations of (A.3), we can obtain the expression for the field in the point of observation $\vec{R}_{1}$ :

$$
\begin{aligned}
\tilde{P}\left(\vec{R}_{1}\right)=\frac{A}{4 \pi} \cdot \int_{S} \frac{e^{-j k\left(\left|\vec{r}-\vec{R}_{1}\right|+\left|\vec{r}-\vec{R}_{0}\right|\right)}}{\left|\vec{r}-\vec{R}_{1}\right|\left|\vec{r}-\vec{R}_{0}\right|} \\
\quad \times\left(\left(j k+\frac{1}{\left|\vec{r}-\vec{R}_{0}\right|}\right) \vec{n}(\vec{r}) \cdot \vec{e}_{0}(\vec{r})\right. \\
\left.+\left(j k+\frac{1}{\left|\vec{r}-\vec{R}_{1}\right|}\right) \vec{n}(\vec{r}) \cdot \vec{e}_{1}(\vec{r})\right) d S .
\end{aligned}
$$

Here $\vec{e}_{0}(\vec{r})$ and $\vec{e}_{1}(\vec{r})$ are the normalized vectors of view from the transmitter and the receiver, respectively,

$$
\vec{e}_{0}(\vec{r})=\frac{\vec{r}-\vec{R}_{0}}{\left|\vec{r}-\vec{R}_{0}\right|}, \quad \vec{e}_{1}(\vec{r})=\frac{\vec{r}-\vec{R}_{1}}{\left|\vec{r}-\vec{R}_{1}\right|} .
$$

Neglecting the small terms of the values $1 /\left|\vec{r}-\vec{R}_{0}\right|$ and $1 /\left|\vec{r}-\vec{R}_{1}\right|$, we can write the final expression for the received field:

$$
\begin{aligned}
& \tilde{P}\left(\vec{R}_{1}\right) \\
& \quad=\frac{j k A}{4 \pi} \cdot \int_{S} \frac{e^{-j k\left(\left|\vec{r}-\vec{R}_{1}\right|+\left|\vec{r}-\vec{R}_{0}\right|\right)}}{\left|\vec{r}-\vec{R}_{1}\right|\left|\vec{r}-\vec{R}_{0}\right|} \vec{n}(\vec{r}) \cdot\left(\vec{e}_{0}(\vec{r})+\vec{e}_{1}(\vec{r})\right) d S .
\end{aligned}
$$

\section{REFERENCES}

[1] R. G. Kouyoumjian and P. H. Pathak, "A uniform geometrical theory of diffraction for an edge in a perfectly conducting surface," Proceedings of the IEEE, vol. 62, no. 11, pp. 1448-1461, 1974.

[2] D. E. Kerr, Ed., Propagation of Short Radio Waves, Peter Peregrinus, London, UK, 1987.

[3] D. A. McNamara, C. W. I. Pistorius, and J. A. G. Malherbe, Introduction to the Uniform Geometrical Theory of Diffraction, Artech House, Boston, Mass, USA, 1990.

[4] W.-M. Boerner, M. B. El-Arini, C.-Y. Chan, and P. M. Mastoris, "Polarization dependence in electromagnetic inverse problems," IEEE Trans. Antennas and Propagation, vol. 29, no. 2, pp. 262-271, 1981.

[5] N. N. Bojarski, "A survey of physical optics inverse scattering identity," IEEE Trans. Antennas and Propagation, vol. 30, no. 5, pp. 980-989, 1982.

[6] A. I. Ladygin and A. A. Lychin, "Physical optics resolving an inverse diffraction task for the rough objects," Electromagnetic Waves \& Electronic Systems, vol. 5, no. 2, pp. 238-251, 2000, (Russian).

[7] Z. Sipus, P.-S. Kildal, R. Leijon, and M. Johansson, "An algorithm for calculating Green's functions of planar, circular cylindrical and spherical multilayer substrates," Applied Computational Electromagnetics Society Journal, vol. 13, no. 3, pp. 243-254, 1998.

[8] G. Galati, M. Ferri, and F. Marti, “(1994) advanced radar techniques for the air transport system: the surface movement miniradar concept," in 1994 IEEE National Telesystems Conference, (NTC '94), San Diego, Calif, USA, May 1994.

[9] G. Galati, M. Ferri, P. Mariano, and F. Marti, "(1994) advanced integrated architecture for ground movements surveillance," in 1995 IEEE International Radar Conference, Alexandria, Va, USA, May 1995.

[10] M. D. Deshpande, C. R. Cockrell, F. B. Beck, E. Vedeler, and M. B. Koch, "Analysis of electromagnetic scattering from irregularly shaped, thin, metallic flat plates," Nasa TP-3361, NASA Langley Research Center, Hampton, Va, USA, September 1993.

[11] E. K. Miller, "A selective survey of computational electromagnetics," IEEE Trans. Antennas and Propagation, vol. 36, no. 9, pp. 1281-1305, 1988.

[12] J. J. H. Wang, Generalized Moment Methods in Electromagnetics, John Wiley \& Sons, NY, USA, 1991.

[13] A. C. Woo, H. T. G. Wang, M. J. Schuh, and M. L. Sanders, “em programmer's notebook-benchmark radar targets for the validation of computational electromagnetics programs," IEEE Antennas and Propagation Magazine, vol. 35, no. 1, pp. 84-89, 1993.

[14] S. M. Rao and D. R. Wilton, "Transient scattering by conducting surfaces of arbitrary shape," IEEE Trans. Antennas and Propagation, vol. 39, no. 1, pp. 56-61, 1991.

[15] N. Y. Zhu and F. M. Landstorfer, "Application of curved parametric triangular and quadrilateral edge elements in the moment method solution of the EFIE," IEEE Microwave and Guided Wave Letters, vol. 3, no. 9, pp. 319-321, 1993. 
[16] M. Born and E. Wolf, Principles of Optics, Pergamon Press, NY, USA, 6th edition, 1980.

[17] E. Hecht, Optics, Addison Wesley, Reading, Mass, USA, 2nd edition, 1987.

[18] J. B. Keller, "Geometrical theory of diffraction," Journal of the Optical Society of America, vol. 52, no. 2, pp. 116-130, 1962.

[19] A. I. Ladygin, Theoretical Bases of the Space Objects Recognition with the Radar Observation, Moscow Aviation Institute, Moscow, 1997, (Russian).

[20] E. Jørgensen, S. Maci, and A. Toccafondi, "Fringe integral equation method for a truncated grounded dielectric slab," IEEE Trans. Antennas and Propagation, vol. 49, no. 8, pp. 1210-1217, 2001.

[21] E. Jørgensen, P. Meincke, and O. Breinbjerg, "An efficient fringe integral equation method for optimizing the antenna location on complex bodies," in IEEE Antennas and Propagation Society Symposium, pp. 584-587, Boston, Mass, USA, July 2001.

[22] J. M. Song, C. C. Lu, and W. C. Chew, "Multilevel fast multipole algorithm for electromagnetic scattering by large complex objects," IEEE Trans. Antennas and Propagation, vol. 45, no. 10, pp. 1488-1493, 1997.

[23] X. Q. Sheng, J. M. Jin, J. Song, W. C. Chew, and C. C. Lu, "Solution of combined field integral equation using multilevel fast multipole algorithm for scattering by homogeneous bodies," IEEE Trans. Antennas and Propagation, vol. 46, no. 11, pp. 1718-1726, 1998.

[24] N. Geng, A. Sullivan, and L. Carin, "Fast multipole method for scattering from an arbitrary PEC target above or buried in a lossy half space," IEEE Trans. Antennas and Propagation, vol. 49, pp. 740-748, May 2001.

[25] Z. Liu, R. J. Adams, and L. Carin, "New MLFMA formulation for closed PEC targets in the vicinity of a half space," submitted to IEEE Trans. Antennas and Propagation.

Yulia V. Zhulina was born in Igarka, Russia. She graduated from the Moscow Physical and Engineering Institute, Moscow, Russia, in 1963. She received her Ph.D. degree in radar engineering from the Moscow Physical and Engineering Institute, Moscow, Russia, in 1968. In 1963, she joined the Radar Engineering Department at Vympel company, where she is currently a Senior Scientist Researcher. She is the coauthor of the book Detecting Moving Objects (Sovetskoye Radio, Moscow, 1980). Her research interests are in image recovery, medical, optical, radar imaging, methods of the "blind deconvolution," recognition with the optical images, and applied mathematical and statistical methods. 